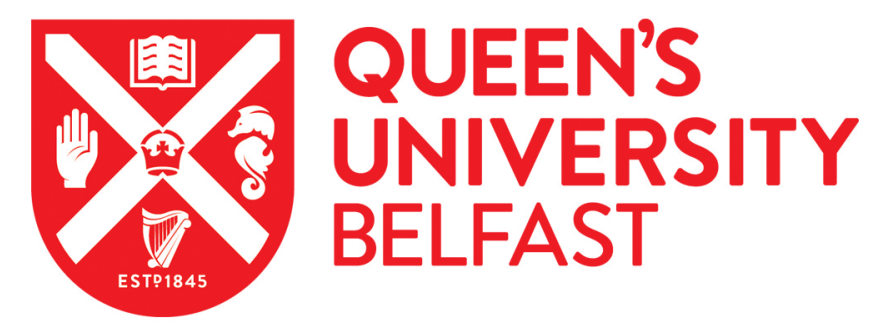

\title{
Multimode Circular Antenna Array for Spatially Encoded Data Transmission
}

Chepala, A., Ding, Y., \& Fusco, V. (2019). Multimode Circular Antenna Array for Spatially Encoded Data Transmission. IEEE Transactions on Antennas and Propagation, 67(6), 3863-3868. [ 10.1109/TAP.2019.2905725 ]. https://doi.org/10.1109/TAP.2019.2905725

Published in:

IEEE Transactions on Antennas and Propagation

Document Version:

Peer reviewed version

Queen's University Belfast - Research Portal:

Link to publication record in Queen's University Belfast Research Portal

Publisher rights

Copyright 2019 IEEE. This work is made available online in accordance with the publisher's policies. Please refer to any applicable terms of use of the publisher.

\section{General rights}

Copyright for the publications made accessible via the Queen's University Belfast Research Portal is retained by the author(s) and / or other copyright owners and it is a condition of accessing these publications that users recognise and abide by the legal requirements associated with these rights.

Take down policy

The Research Portal is Queen's institutional repository that provides access to Queen's research output. Every effort has been made to ensure that content in the Research Portal does not infringe any person's rights, or applicable UK laws. If you discover content in the Research Portal that you believe breaches copyright or violates any law, please contact openaccess@qub.ac.uk. 


\title{
Multi-mode Circular Antenna Array for Spatially Encoded Data Transmission
}

\author{
Anil Chepala, Yuan Ding and Vincent Fusco, Fellow, IEEE
}

\begin{abstract}
We present a new technique for data transmission by wireless. We show how a circular array can have each of its major radiating modes orthogonally encoded with data. These data impressed radiating modes are concurrently excited, and then spatially combined, in order to give a structured far-field radiation pattern. Also discussed is how a receiver can recover these orthogonal data streams. A proof of principle Multi-Mode Circular Array (MMCA) is designed, simulated and tested at 900 MHz.
\end{abstract}

Index Terms-Circular Array, Multi-Mode Antenna Orthogonal Coding.

\section{INTRODUCTION}

Circular antenna arrays have been used for many years in angle of arrival applications [1]-[7] . The main advantage of circular arrays is their far-field radiation pattern symmetry, which, unlike in linear arrays, can be exploited to scan a beam through $360^{\circ}$ without variation in gain and pattern shape. The purpose of this paper is to demonstrate how a multi-mode circular array can be used for transmitting parallel data by encoding, then combining the orthogonal radiating modes. The approach suggests an additional degree of freedom, which augments existing techniques for physical-layer secured wireless data transmission, e. g. [8].

The theoretical and simulation aspects of the architecture of an MMCA are discussed in Section II along with their Envelope Correlation Coefficients (ECC). The effective bandwidth definitions particular to MMCA usage proposed in this paper and theoretical ripple factor are discussed in Section III. Spatial data encoding with Walsh codes and demodulation of the sampled patterns in the presence of AWGN are discussed in Section IV, while the simulated results based on real active array patterns are presented in Section V. In Section VI, we present the simulated BER results and finally, Section VII concludes the paper.

\section{MULTI-MODE CIRCULAR ARRAY (MMCA)}

The classical architecture of the circular array transmitter, [1] is shown in Fig. 1, which shows a circular dipole array

This work was supported by Queens University of Belfast Studentship, and the UK Engineering and Physical Science Research Council (EPSRC) under grants EP/P000673/1 and EP/NO20391/1.

A. Chepala and V. Fusco are with The Centre for Wireless Innovation (CWI), The Institute of Electronics, Communications and Information Technology (ECIT), School of Electronics, Electrical Engineering and Computer Science (EEECS), Queen's University Belfast, Belfast BT3 9DT, United Kingdom. (emails: achepala01@qub.ac.uk and v.fusco@ecit.qub.ac.uk)

Y. Ding is with the Institute of Sensors, Signals and Systems (ISSS), Heriot-Watt University, Edinburgh EH14 4AS, United Kingdom. (email: yuan.ding@hw.ac.uk)

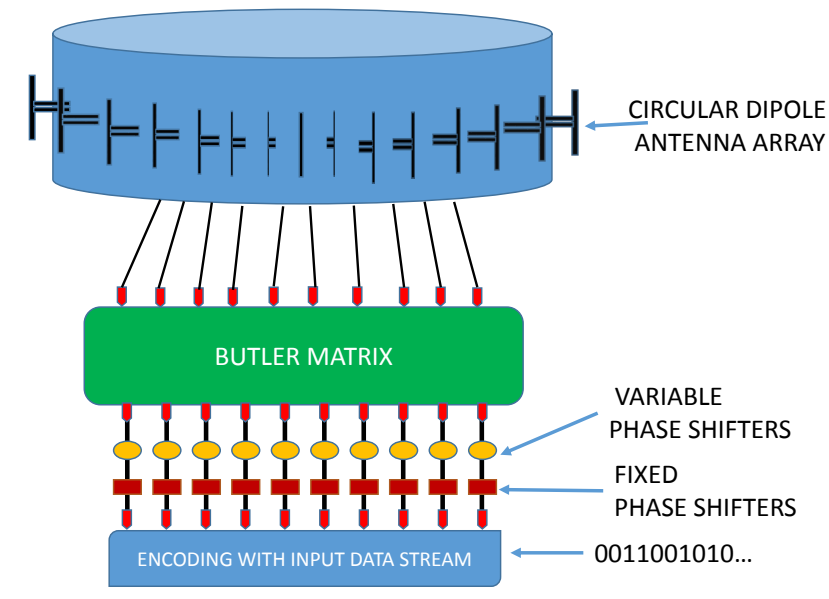

Fig. 1. Multi-mode circular array (MMCA) architecture.

backed with a quarter-wavelength spaced metal reflector. Classically a Butler Matrix (BM), [9], is used to generate the required phase mode excitation vectors. Fixed and variable phase shifters energised by a single source are employed for mode alignment and beam rotation, respectively as discussed in [4]-[7].

The operation principle of a multimode circular array can be understood by considering the arrays current distribution [1][3]. When this distribution is expanded as a Fourier series, each term represents a current mode. The radiation pattern of each mode has the same form as the current mode itself, and these modes are themselves the Fourier components of the radiation pattern of the original distribution.

Consequently, the horizontal far-field directional pattern $F(\phi)$ can be mathematically expressed as a complex Fourier series that is a function of both amplitude and phase.(see Eq.(1)).

$$
F(\phi)=\sum_{-M}^{M} C_{m} e^{j m \phi}
$$

where,

$$
C_{m}=\int_{0}^{2 \pi} F(\phi) e^{-j m \phi}
$$

$\phi=$ azimuth angle and $m=$ mode number

Each of these modes in Eq.(2) can be excited independently e.g. by using a Butler Matrix (BM) [9]. 


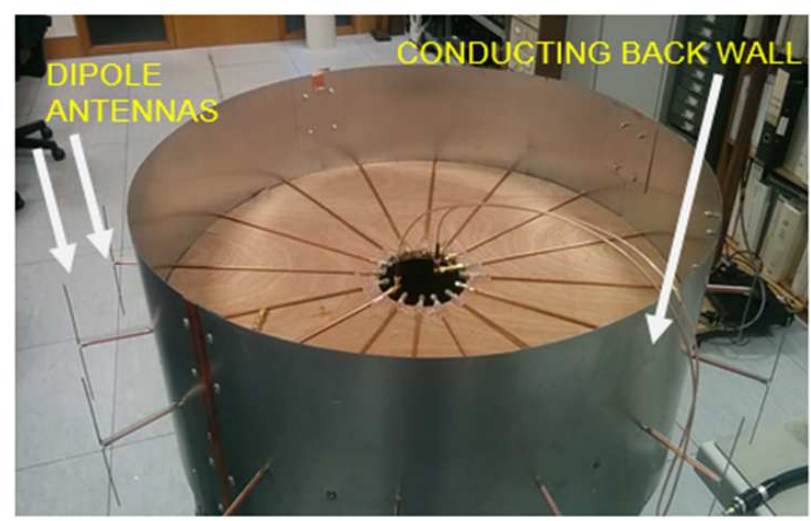

Fig. 2. 16-element MMCA prototype.

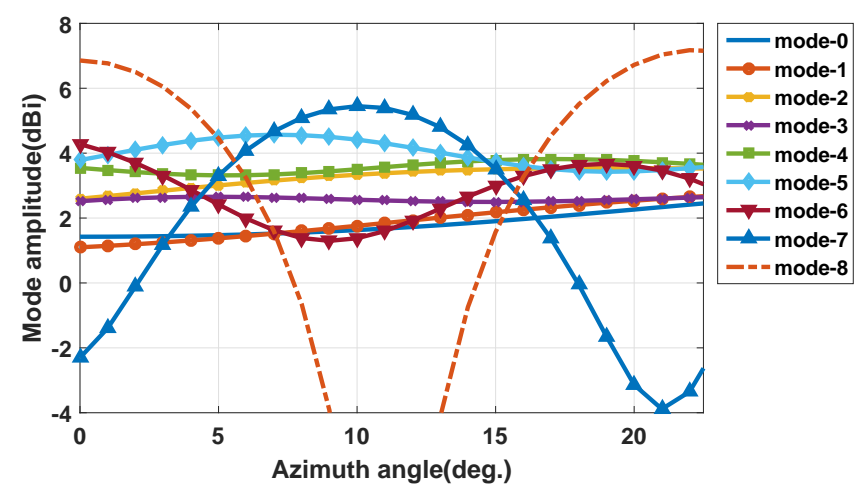

Fig. 3. MMCA simulated far-field mode amplitude response at $0.9 \mathrm{GHz}$ for the first eight modes of the structure.

If $B_{K} e^{j \beta_{k}}$ is the current applied to the $K-t h$ input port of an $N \times N \mathrm{BM}$, the resultant $A_{J} e^{j \psi_{J}}$ on the $J-t h$ radiating element in an array of $N$ elements is given by is given by

$$
A_{J} e^{j \psi_{J}}=\frac{1}{\sqrt{N}} B_{K} e^{j \beta_{k}} e^{j K J(2 \pi / N)}
$$

When many inputs are simultaneously excited, the output current distribution is the summation over $K$ for $N$ element array. The far-field radiation pattern, assuming the approximate pattern of dipole $D(\phi)$ as $(1+\cos \phi)$ is given by

$$
\begin{array}{r}
E(\phi)=\sum_{J=1}^{N}\left[\sum_{K=1}^{N} B_{K} e^{j \beta_{k}} e^{j K J(2 \pi / N)}\right] \\
\cdot\left[D\left(\phi-\alpha_{J}\right) e^{j(2 \pi R / \lambda) \cos \left(\phi-\alpha_{J}\right)}\right]
\end{array}
$$

Where $\lambda$ is the wavelength of operation, $\beta$ is the wavenumber and $R$ the radius of the circular array. $\alpha_{J}$ is the element angular location (with $N$ equally spaced elements at $\alpha_{J}=$ $J(2 \pi / N)$ when $J=1,2, \ldots N)$. Referring to the circular array center, the relative phase of the $J-t h$ element in this case would be $(2 \pi R / \lambda) \cos \left(\phi-\alpha_{J}\right)$.

In the work we present here, a new development is reported. Here we encode each of the principle MMCA modes with orthogonal Walsh Binary Phase Shift Key (BPSK) modulated data in order to form a data word that is spatially decoded onto the far-field pattern of MMCA. In doing so we create an azimuthally structured radiation pattern that is uniquely defined by the characteristic shape of the far-field radiating pattern that results from mode summation and imprinted data word.

Fig. 2 shows the prototype MMCA as experimentally characterized. Each of the 16 radiating elements of the MMCA are designed to operate at $0.9 \mathrm{GHz}$. The MMCA consists of 16 half-wavelength dipole antennas each fed by a quarterwavelength balun and arranged around a circle of diameter $848 \mathrm{~mm}$. The metallic cylinder diameter is $680 \mathrm{~mm}$, height $300 \mathrm{~mm}$ and thickness $2 \mathrm{~mm}$. The dipoles are separated from the metallic cylinder by a distance of $83 \mathrm{~mm}$, which is approximately one-quarter wavelength at the array operating frequency, and the dipoles are uniformly separated by $22.5^{\circ}$.

The resulting mode amplitude patterns for the first eight modes of the structure are shown in Fig. 3. It is noted that not all the modes radiate with equal strength, and that the variation of the phases of modes from 0 to 6 is within \pm 1.6 $\mathrm{dB}$. The modes exhibit linear phase response $(\leq \pm 1.06)$ up to modes \pm 4 (see Table I (b)). Hence, in the rest of this paper only mode up to \pm 4 are used for encoding. Following [7], these modes are aligned to a common phase reference of $0^{\circ}$ at azimuth angle of $0^{\circ}$ using the fixed phase shifters shown in Fig. 1.

Next, we check the orthogonality between the far-field patterns associated with the modes, which is a necessary requirement for minimizing spatial cross coupling between imprinted data streams. This is done by computing the envelope correlation coefficient (ECC) represented by $\rho_{e},[10]$, of each of the MMCA modes. The 3D far-field patterns for each of the modes from the simulation are recorded over the frequency band from $0.4 \mathrm{GHz}$ to $1.6 \mathrm{GHz}$. $\rho_{e}=1.0$ means patterns fully correlated and $\rho_{e}=0$ implies patterns are uncorrelated i.e. orthogonal to each other. The maximum $\rho_{e}$ in the frequency band over all modes up to \pm 4 is plotted as shown in Fig. 4. These results suggest that $22 \%$ correlation bandwidth should be achievable for $\rho_{e} \leq 0.02$ and $13 \%$ for $\rho_{e} \leq 0.01$.

\section{BANDWIDTH ANALYSIS OF MMCA}

To access the bandwidth performance of the 16-element MMCA several parameters are considered: (A) The ECC bandwidth, (B) the impedance bandwidth, (C) the peak-topeak variation of amplitude and RMS phase deviation of modes over frequency, (D) the total radiated power of modes over frequency, and (E) theoretical ripple factor as function of mode number.

\section{A. ECC Bandwidth}

From Fig. 5, it can be seen that the mode ECC exhibits a faster rise in its value as frequencies are reduced below the centre operating frequency of the array and rise gradually for frequencies above the centre frequency. This is illustrated in Fig. 4, which indicates that as the operating frequency is reduced the mode patterns become more similar in structure, and therefore ECC increases. When the frequency increases more modes exist and therefore ECC remains low due to richer spatial diversity. 


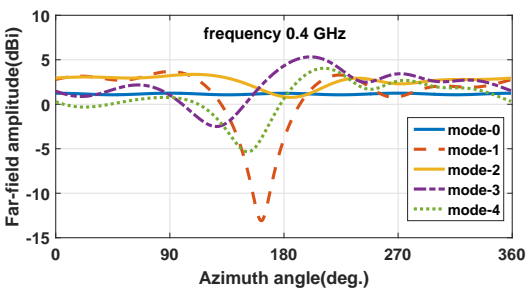

(a)

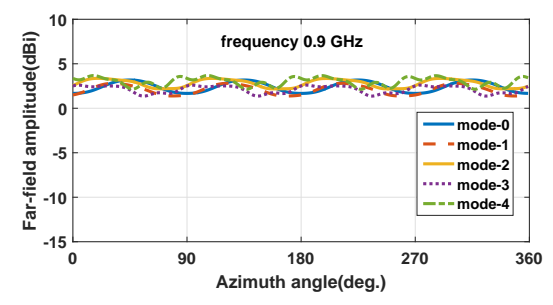

(b)

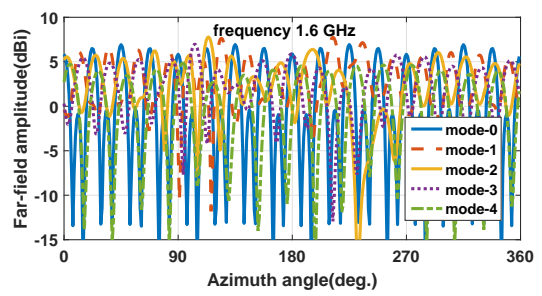

(c)

Fig. 4. Simulated mode patterns (modes 0 to 4) showing spatial diversity with frequency. (a) $0.4 \mathrm{GHz}$, (b) $0.9 \mathrm{GHz}$ and (c) $1.6 \mathrm{GHz}$.

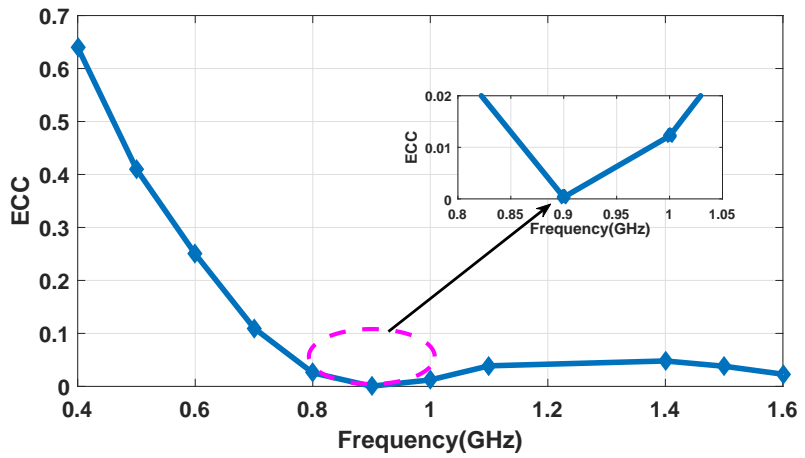

Fig. 5. Maximum ECC of modes versus frequency.

\section{B. Impedance Bandwidth}

The return loss of the radiating elements of the array varies with mode excitation number. As higher order modes are excited, ripple is introduced into the dipole input impedance response. For example, Fig. 6 shows the reflection coefficient of dipole-1 (with the other dipoles terminated) for the first five modes excited on the array. As can be seen ripple in the reflection coefficient increases with mode number. The higher order modes (above mode 4) are subjected to more variation and are generally worse at the higher frequency end than at the lower frequency. These results show that modes up to four can be used for transmission over a band from $0.86 \mathrm{GHz}$ to $1.2 \mathrm{GHz}$ since return loss is less than $10 \mathrm{~dB}$.

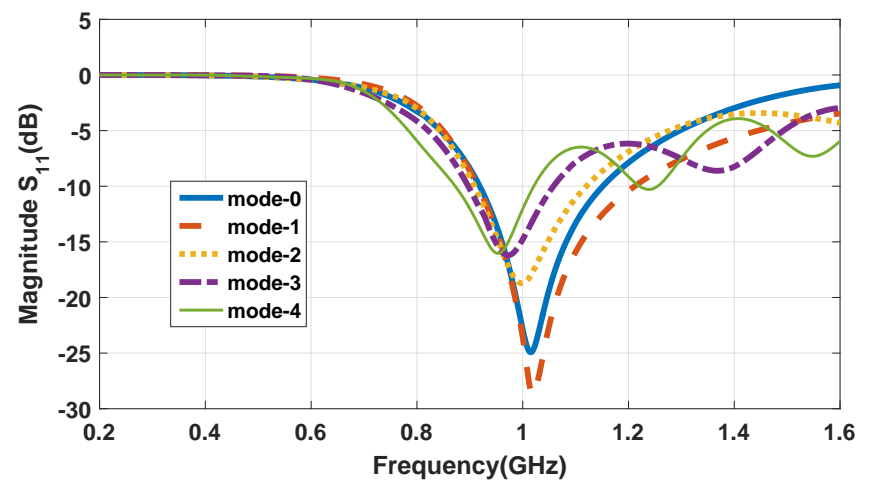

Fig. 6. Reflection coefficients of dipole-1 in MMCA for different mode excitations with other dipoles terminated.
TABLE I

MODE RIPPLE AND PHASE DEVIATION WITH FREQUENCY

\begin{tabular}{|c||c|c|c|c|c|c|c|}
\hline $\begin{array}{c}\text { Frequency(GHz) } \\
\text { Mode Number }\end{array}$ & $\mathbf{0 . 8 0}$ & $\mathbf{0 . 8 5}$ & $\mathbf{0 . 9 0}$ & $\mathbf{0 . 9 5}$ & $\mathbf{1 . 0 0}$ & $\mathbf{1 . 1 0}$ & $\mathbf{1 . 2 0}$ \\
\hline $\mathbf{0}$ & 2.50 & 1.77 & 1.87 & 1.02 & 1.14 & 0.86 & 1.04 \\
\hline $\mathbf{1}$ & 4.35 & 2.52 & 2.05 & 1.90 & 2.41 & 6.34 & 13.6 \\
\hline $\mathbf{2}$ & 8.25 & 4.05 & 1.71 & 3.23 & 7.07 & 17.4 & 25.8 \\
\hline $\mathbf{3}$ & 13.6 & 6.10 & 1.64 & 4.60 & 11.1 & 24.4 & 8.02 \\
\hline $\mathbf{4}$ & 23.5 & 6.60 & 1.92 & 6.70 & 26.7 & 11.3 & 24.2 \\
\hline
\end{tabular}

(a) Maximum Ripple in $\mathrm{dB}$

\begin{tabular}{|c||c|c|c|c|c|c|c|}
\hline $\begin{array}{c}\text { Frequency(GHz) } \\
\text { Mode Number }\end{array}$ & $\mathbf{0 . 8 0}$ & $\mathbf{0 . 8 5}$ & $\mathbf{0 . 9 0}$ & $\mathbf{0 . 9 5}$ & $\mathbf{1 . 0 0}$ & $\mathbf{1 . 1 0}$ & $\mathbf{1 . 2 0}$ \\
\hline $\mathbf{0}$ & 0.003 & 0.35 & 1.39 & 1.99 & 2.19 & 2.88 & 2.30 \\
\hline $\mathbf{1}$ & 8.57 & 4.50 & 0.18 & 5.12 & 9.56 & 18.5 & 30.2 \\
\hline $\mathbf{2}$ & 19.3 & 9.65 & 0.15 & 8.83 & 18.7 & 41.7 & 64.6 \\
\hline $\mathbf{3}$ & 29.5 & 14.3 & 0.96 & 14.3 & 28.1 & 31.1 & 16.3 \\
\hline $\mathbf{4}$ & 41.7 & 19.4 & 1.06 & 18.9 & 39.5 & 18.0 & 140 \\
\hline
\end{tabular}

(b) RMS phase deviation in degree

C. The peak-to-peak variation of amplitude and rms phase deviation of modes over frequency

Here we examine mode maximum amplitude ripple and root mean square (RMS) phase variation defined as RMS variation of phase about a linear fit of the unwrapped phase plot as a function of azimuth angle. The amplitude ripple computed over azimuth angle $0^{\circ}$ to $360^{\circ}$ for different frequencies is shown in Table I. It can be observed that at the centre operating frequency of the array the ripple is below $2.0 \mathrm{~dB}$ up to mode4 , after which it increases rapidly. Similarly, amplitude ripple increases very quickly away from the centre frequency. The RMS phase deviations follow a similar pattern.

\section{The Total Radiated Power of Modes over Frequency}

The total radiated power (TRP) associated with each mode over frequency is next examined. The total radiated power relative to mode-0 are shown in the Fig. 7. Here it can be seen that different modes radiate at different power levels over the band of frequencies. It is observed that modes up to four radiate most effectively (above 0.8 ) from $0.85 \mathrm{GHz}$ to 1.1 $\mathrm{GHz}$. 


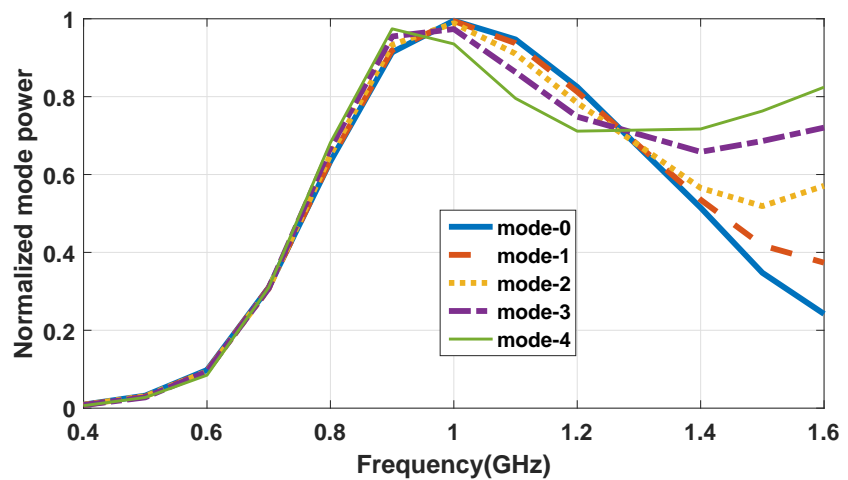

Fig. 7. Total radiated power of all modes vs frequency

\section{E. Theoretical Ripple Factor $\left(R_{m}\right)$}

The theoretical ripple factor $\left(R_{m}\right)$ for a circular directional modulation transmitter array was derived from the fundamental equations of mode patterns for 32 elements in [8]. By applying this theory, we derive the ripple factor for 16element MMCA shown in Fig. 2. The ripple factor $\left(R_{m}\right)$ is calculated as per Eq. (5), [8], where $P_{m}$ and $Q_{m}$ are the desired mode magnitude and the distortion magnitude respectively. The ripple factor in $d B$ is plotted against mode order $m$ for the 16-element MMCA as shown in Fig. 8.

$$
R_{m}=20 \times \log _{10}\left|\frac{P_{m}+Q_{m}}{P_{m}-Q_{m}}\right|
$$

From the theoretical graphs plotted for the MMCA at 0.9 $\mathrm{GHz}$, it is evident that the structure supports modes with ripple factor less than $1 \mathrm{~dB}$ for modes less than 5 . With increase in order number $m$, the distortion term increases, thereby resulting in increased $R_{m}$.

From the various bandwidth analysis on the MMCA conducted in this section, results such as return loss $\leq 10 \mathrm{~dB}$, amplitude ripple $\leq 2 \mathrm{~dB}$ and TRP $>0.7$, it can be concluded that modes up to \pm 4 can be used effectively and efficiently for modulation encoding over a band from 0.85 to $1.2 \mathrm{GHz}$. The encoding of these modes and the receiving methodology is discussed in the following sections.

\section{DATA ENCODING AND RECEIVER PATTERN CAPTURE}

Next we describe the process by which we encode each of the \pm 4 principle modes with orthogonal binary 8-bit Walsh (temporally orthogonal) coded BPSK signals. Mode-0 is used as a phase reference (can also be used to transmit data). One bit of which is imprinted onto each of the \pm 4 modes of the MMCA. BPSK modulation is performed by using the variable one-bit phase shifters shown in Fig. 1, each connected to the corresponding bit of the parallel bit stream. The input to the BM (Eq. (3)) circuit ports is the BPSK signal, with its outputs connected to the 16 antenna array ports of the MMCA.

Using the approach described in [1], the beam pattern is electronically scanned in the azimuth plane by using variable phase shifters in Fig. 1. Here we take advantage of MMCA

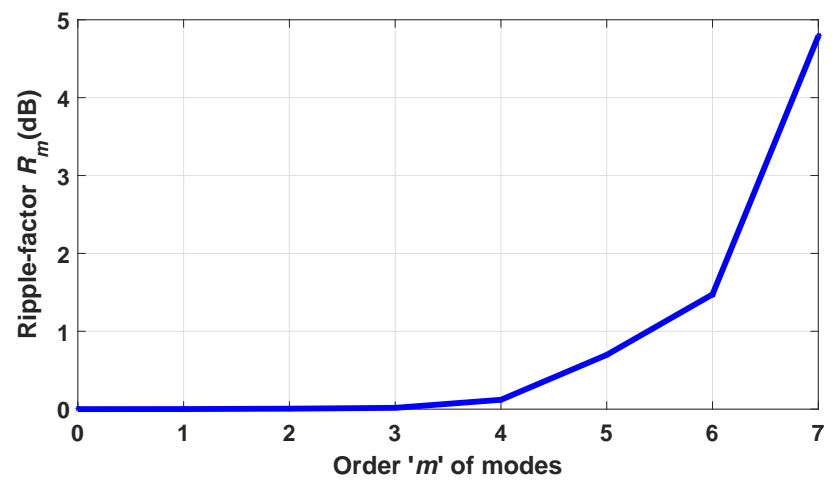

Fig. 8. MMCA ripple factor versus mode order $m$ at $0.9 \mathrm{GHz}$.

symmetry, whereby we can scan a beam through a full $360^{\circ}$ without variations in gain and pattern shape [1], [2].

Here the 8-bit data pattern encoded in parallel to the modes \pm 4 , applied to the BM input ports is held, and the resultant MMCA pattern rotated through $360^{\circ}$. This ensures that the receiver gets sight of the complete pattern for a given input data vector. The receiver can be located in the azimuth plane anywhere in the far-field in an unobstructed link. Therefore, as a consequence of this application of data streams to individual modes, we create an azimuthally structured far-field radiation pattern that is uniquely defined by the mode summation and is imprinted with the signature of the data stream.

In order for a single distant receiver arbitrarily positioned at any azimuthal position to decode this imprinted data from the structure of the far-field patterns shown in Fig. 11, two things must occur. First, a phase reference has to be established. This is achieved by using mode -0 in Fig. 3 since it has a constant amplitude and phase over $360^{\circ}$ in azimuth.

For the purposes of biterrorrate (BER) simulation, complex AdditiveWhiteGaussianNoise (AWGN) is added to the transmitted azimuth pattern, and the resultant signal is then decoded using a correlation receiver, [11]. The information now encoded onto the MMCA far-field patterns, is demodulated at the receiver at different sampling rates. A correlation receiver principle based on the Fourier mode structure of the MMCA, is used in the decoding algorithm, [11].

The decomposition of the far-field radiation pattern follows from Eq. (2) as, $r_{k}$ is the received field pattern which consists of both the information signal $s_{m}(t)$ and the noise signal $n(t)$. This signal is now separated into its components by correlating with the respective mode component $f_{k}(t)$ as shown in Eq. (6).

$$
\begin{array}{r}
r_{k}=\int_{0}^{T} r(t) f_{k}^{*}(t) d t=\int_{0}^{T}\left[s_{m}(t)+n(t)\right] f_{k}^{*}(t) d t \\
=\int_{0}^{T} s_{m}(t) f_{k}(t) d t+\int_{0}^{T} n(t) f_{k}^{*}(t) d t \\
=s_{m k}+n_{k}, \quad 1 \leq k \leq N \\
s_{m}(t)=\int_{0}^{T} r(t) f_{k}^{*}(t) d t
\end{array}
$$




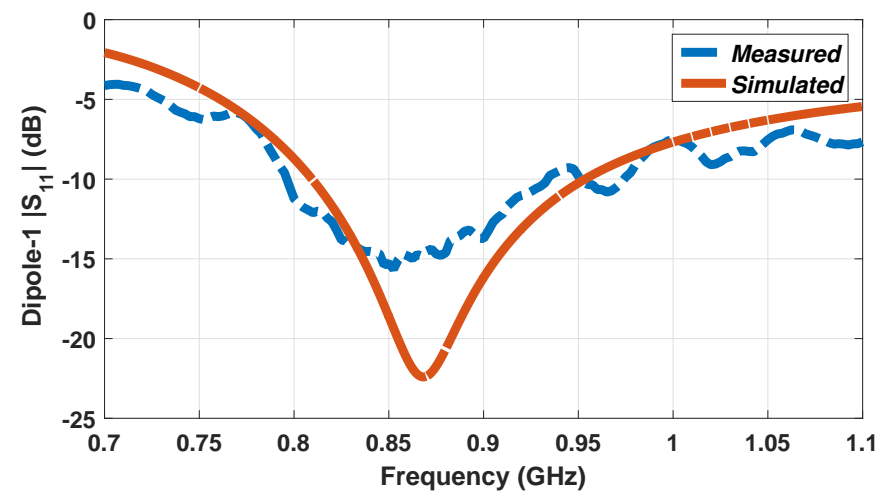

Fig. 9. MMCA dipole reflection coefficient.

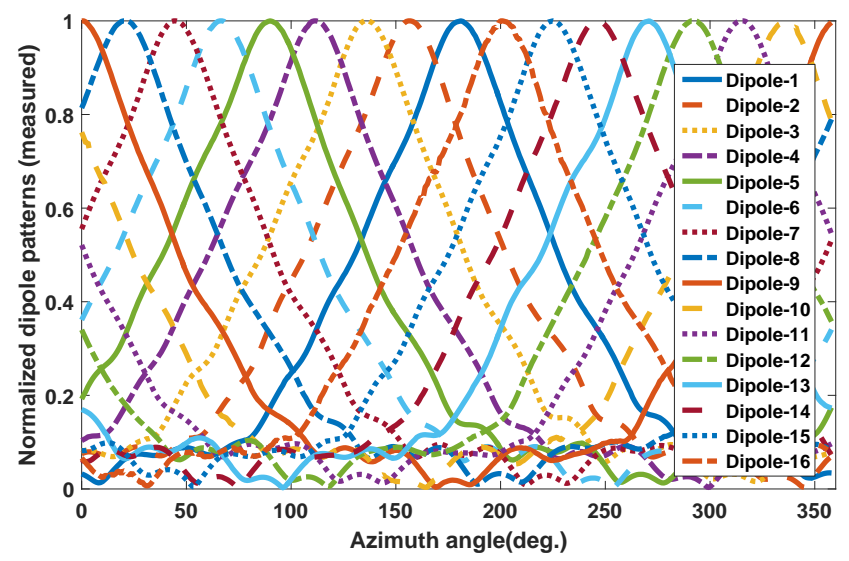

Fig. 10. Measured radiation pattern of dipoles in MMCA at $0.9 \mathrm{GHz}$.

The resulting fuction is then integrated over the azimuth angle $t$ to obtain a value which represents the equivalent of corresponding data bit. This demodulated data Eq. (7) is then decoded by passing through Maximum Likelhood (ML) detector [11]. The input 8-bit data patterns are varied randomly and the corresponding BER is evaluated for different signal - to - noise ratio (SNR) and receiver spatial pattern sampling rates. The results can then be compared to the conventional (Nyquist) BER Vs SNR curve for BPSK modulation.

\section{MMCA PROTOTYPE}

Using the experimental arrangement in Fig. 2, the reflection coefficient of all the 16 dipoles was found to be almost identical; a sample dipole $\left|S_{11}\right|$ is shown in the Fig. 9. The reflection coefficient is below $-10 \mathrm{~dB}$ in the frequency band from $0.8 \mathrm{GHz}$ to $0.93 \mathrm{GHz}$. The measured mutual coupling $\left|S_{21}\right|$ between the nearest elements in all cases was less than $18 \mathrm{~dB}$. The far-field active patterns, [1], of each dipole were measured in an anechoic chamber. Virtually identical far-field patterns were obtained for every element of the array as shown in Fig. 10.

The far-field patterns for all Walsh code inputs are evaluated from the measured dipole patterns. Examples of the measured amplitude, after normalisation, for an input Walsh codes 00000000, and 0101-0101, is shown in Fig. 11a and 11b respectively and compared with those obtained using theoretical

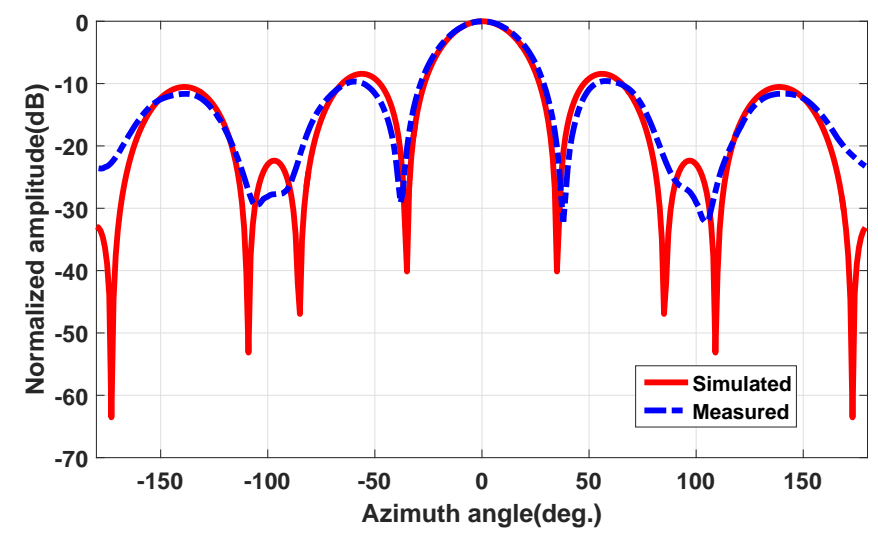

(a)

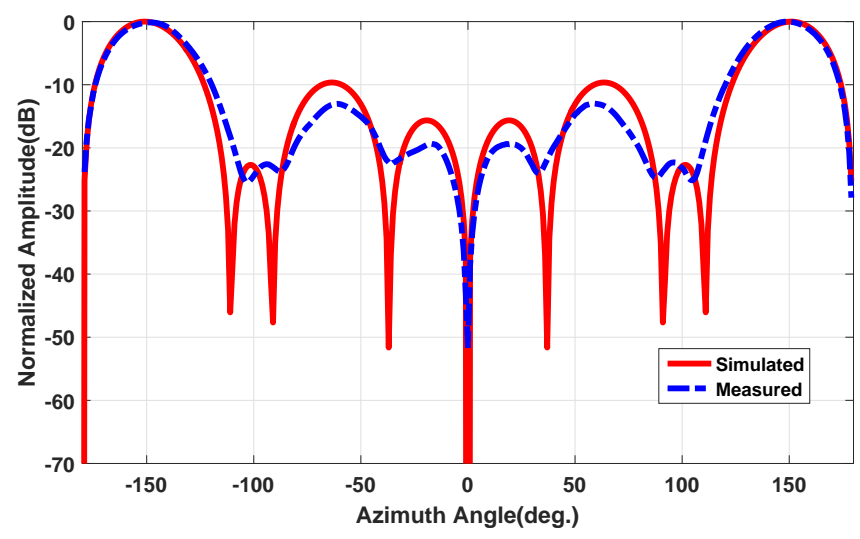

(b)

Fig. 11. Example Walsh encoded far-field patterns. (a) 0000-0000. (b) 01010101.

cosine dipole pattern distributions. Equivalent comparisons exist for all possible Walsh codes.

\section{SIMULATED BER RESULTS}

Using the MMCA active patterns, Fig. 12 shows the computed BER Vs SNR curve after both amplitude and phase noise is accounted for. The spatial distance between the samples in degrees or the sampling interval is given by $D s$, e.g. $D s=30$, means that pattern is sampled at every $30^{\circ}$, i.e. 12 samples per composite pattern revolution. In this case, the BER of the system is close to that of a conventional BPSK system as evident from the BER graph ( see dashed black curve in Fig. 12). Over-sampling improves the BER recovery statistics while under-sampling leads to performance degradation. Taking mode -0 as phase reference simplifies data acquisition since the receiver can be placed at any arbitrary range with respect to the MMCA. It is noted that better BER can be achieved with higher sampling rates at the expense of lower data throughput rates due to the time needed to scan the entire pattern through $360^{\circ}$.

\section{CONCLUSION}

We have shown that it is possible to use an MMCA in order to transmit orthogonal data streams comprised of parallel data 


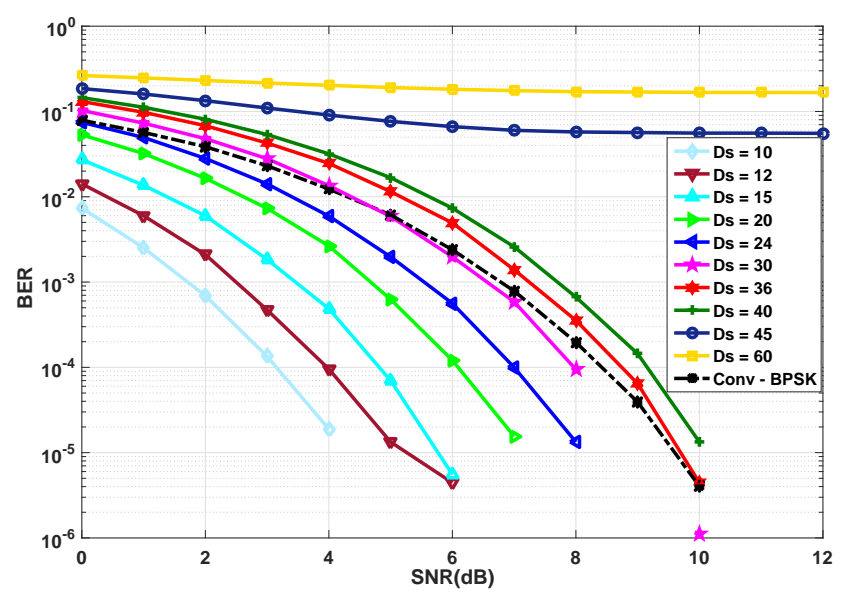

Fig. 12. Simulated BER recovery with mode- 0 as phase reference.

words by direct modulation of the MMCA current modes. This is achieved by spatially encoding the data into the entire far field structure of the transmitted radiated pattern. In order for a classical receiver to decode the transmitted data, it is necessary for it to accumulate the radiated far-field pattern and then apply simple Fourier mode deconstruction. Using the active patterns measured for a 16-element MMCA it was shown that with appropriate sampling of the far-field pattern radiated by the MMCA, that encoded data can be recovered with low BER even at moderate SNRs. The approach suggested offers the possibility for a new type of wireless transmission system wherein an additional element of physical layer security is added through entire pattern interrogation. Such a system may find application in for example a secure line-of-sight point-topoint communications link.

\section{ACKNOWLEDGMENTS}

The authors would like to acknowledge the support given by Centre for Wireless Innovation (CWI) Scholarship at Queens University Belfast and to the EPSRC under the grants EP/P000673/1, EP/NO20391/1. The authors would also like to thank Mr. Kieran Rainey for fabrication and testing of the MMCA.

\section{REFERENCES}

[1] B. Sheleg, "A matrix-fed circular array for continuous scanning," Proc. IEEE, vol. 56, no. 11, pp. 2016-2027, Nov. 1968.

[2] I. D. Longstaff, P. E. K. Chow, and D. E. N. Davies, "Directional properties of circular arrays," Proc. IEE, vol. 114, no. 6, pp. 713-718, Jun. 1967

[3] W. R. Lepage, C. S. Roys, and S. Seely, "Radiation from circular current sheets," Proc. IRE, vol. 38, no. 9, pp. 1069-1072, Sept. 1950.

[4] D. E. N. Davies and B. S. McCartney, "Cylindrical arrays with electronic beam scanning," Proc. IEEE, vol. 112, no. 3, pp. 497-505, Mar. 1965.

[5] R. G. Fenby, "Limitations on directional patterns of phase-compensated circular arrays," Radio Electronics Engr., vol. 30, no. 4, pp. 206-222, Oct. 1965.

[6] R. G. Fenby and D. E. N. Davies, "Circular array providing fast 360 electronic beam rotation," Proc. IEE, vol. 115, no. 1, pp. 78-86, Jan. 1968.

[7] T. Rahimm, "Directional pattern synthesis in circular arrays of directional antennas."
[8] Y. Ding, V. Fusco, and A. Chepala, "Circular directional modulation transmitter array," IET Microw. Antennas Propaga., vol. 11, no. 13, pp. 1909-1917, Oct. 2017.

[9] H. Moody, "The systematic design of the butler matrix," IEEE Transactions on Antennas and Propagation, vol. 12, no. 6, pp. 786-788, Nov 1964.

[10] A. Ourir, K. Rachedi, D. T. Phan-Huy, C. Leray, and J. de Rosny, "Compact reconfigurable antenna with radiation pattern diversity for spatial modulation," in 2017 11th European Conference on Antennas and Propagation (EUCAP), March 2017, pp. 3038-3043.

[11] R. Schober, Optimum reception in additive white gaussian noise AWGN, chapter 4, Detection and estimation of signals in noise". University of British Columbia, Vancouver, Aug. 2010. [Online]. Available: http://courses.washigton.edu/ee506/handouts/slides1.pdf

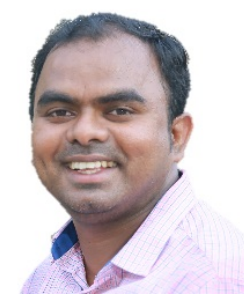

Anil Chepala received his Bachelor's degree in Electronics and Communications Engineering from Andhra University, Visakhapatnam in 2005. He received his Master's degree in Radio Frequency design and technology from Indian Institute of Technology Delhi, New Delhi in 2012. Currently he is pursuing his $\mathrm{Ph} . \mathrm{D}$. in multimode antenna arrays from the Queen's University of Belfast (QUB) since 2016.

He joined Defence Research and Development Organisation(DRDO), Ministry of Defence, Govt. of India, as Scientist in May 2007 and has been serving since then in various activities like antenna and radome design for airborne applications, antenna, radome and RCS calibration and measurements both in near and far field. He was actively involved in successful installation of Near-field test facility at RCI lab in Hyderabad, where he is currently serving as Scientist-D. His research interests include antenna and radome design, beamforming techniques, retroarrays and RCS mitigation techniques for RF stealth using FSS.

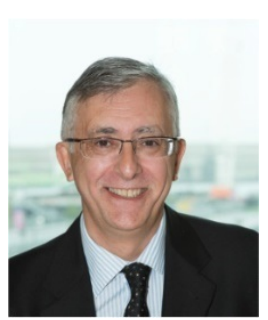

Vincent F. Fusco (S'82-M'82-SM'96-F'04) received the bachelor's (Hons.) degree in electrical and electronic engineering, the Ph.D. degree in microwave electronics, and the D.Sc. degree from Queen's University Belfast (QUB), Belfast, U.K., in 1979, 1982, and 2000, respectively.

His work was focused on advanced front-end architectures with enhanced functionality. He is Chief Technology Officer (CTO) of the ECIT at QUB. He has authored over 450 scientific papers in major journals and in referred international conferences. He has authored two textbooks, holds patents related to self-tracking antennas, and has contributed invited papers and book chapters. His current research interests include active antenna and front-end MMIC techniques.

Prof. Fusco is a fellow of the Institute of Electrical and Electronics Engineers, the Institution of Engineering and Technology, the Royal Academy of Engineers, and the Royal Irish Academy. In 2012, he received the IET Senior Achievement Award and the Mountbatten Medal. He serves on the Technical Program Committee of various international conferences, including the European Microwave Conference 


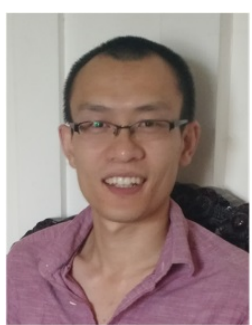

Yuan Ding received his Bachelor's degree from Beihang University (BUAA), Beijing, China, in 2004, received his Master's degree from Tsinghua University, Beijing, China, in 2007, and received his Ph.D. degree from Queens University of Belfast, Belfast, UK, in 2014, all in Electronic Engineering.

$\mathrm{He}$ was a radio frequency (RF) Engineer in Motorola R\&D Centre (Beijing, China) from 2007 to 2009, before joining Freescale Semiconductor Inc. (Beijing, China) as an RF Field Application Engineer, responsible for high power base-station amplifier design, from 2009 to 2011. He is now an Assistant Professor at the Institute of Sensors, Signals and Systems (ISSS) in Heriot-Watt University, Edinburgh, UK. His research interests are in antenna array, physical layer security, and $5 \mathrm{G}$ related areas.

Dr. Ding was the recipient of the IET Best Student Paper Award at LAPC 2013 and the recipient of the Young Scientists Awards in General Assembly and Scientific Symposium (GASS), 2014 XXXIst URSI. 